\title{
Effects of permanent acupuncture of YNSA (Yamamoto new scalp acupuncture) on quality of life in patients suffering from post-stroke syndrome
}

\begin{abstract}
Objectives: The aim of our newer study involving more numbers of the patient was to investigate whether different form acupuncture, Yamamoto's New Scalp Acupuncture (YNSA) affects, to standard post-stroke motor rehabilitation elevating QoL of patients with higher self-management.

Design: A prospective, randomized control research was carried out in an outpatient stroke rehabilitation unit with day hospital service again enrolled 520cases. After inclusion, patients were stratified into control group and acupuncture group, randomly. All of the stratified patients participated in gymnastic exercises and conservative pharmacological treatment according to western rehabilitation protocol.
\end{abstract}

Outcome measures: BI (The Barthel Index), RI (Rivermead Scale Index), the SP-36 questionnaire and VAS were used to follow the results of treatment.

Results: The Barthel Index is increased from $4 \pm 2$ to $95 \pm 4$ in the acupuncture group. This index also increased in the control group (from $4 \pm 2$ to $75 \pm 4$ ), but the changes were significantly less than in the acupuncture group. In the acupuncture group, all the sensory, motor, and functional scores, QoL was improved significantly during the examination period and until three years after injury. We also found a significant spontaneous recovery during the 3-year follow-up, but the YNSA treatment facilitated the functional recovery not only in moving but generally in cognitive functions and memory functions. The complementary treatment improved moving function and more flexible joints and ligaments were observed in comparison to the patients' condition before treatment.

Conclusions: The summarized data suggest that the YNSA acupuncture is might be a useful complementary method to treat residual symptoms of stroke patients and enhance their quality of life, to achieve higher self-management level. As soon as possible after the stroke, this method should be started if cardio-pulmonary condition of a patient is balanced and allowed it. This study was a reproduction of our earlier research with more participants to allowed us to prove advantages of the early application of permanent acupuncture treatment.

Keywords: stroke, post-stroke syndrome, permanent acupuncture, rehabilitation, quality of life (QoL), VAS
Volume II Issue I - 2018

\author{
István Molnár,' Agnes Máté, I Henrik Szőke, ${ }^{2}$ \\ Gabriella Hegyi ${ }^{2}$ \\ 'University of Pécs, Hungary \\ ${ }^{2}$ Department of Complementary Medicine, University of Pécs, \\ Hungary
}

Correspondence: Hegyi Gabriella, Department of Complementary Medicine, Faculty of Health Sciences, University of Pécs, Pécs, Hungary, Email drhegyi@hu.inter.net

Received: February 10, 2018 | Published: February 21, 2018
Abbreviations: BI, barthel index; RMI, rivermead index; VAS, visual analogue scale; YNSA, yamamoto new scalp acupuncture; LTP, long-term-potentiation, LU, lung, LI, large intestine, KI, kidney, BL, bladder; HT, heart; SI, small intestine; LR, liver; GB, gall-bladder; PC, pericardium; TB, triple burner; SP, spleen-pancreas; ST, stomach

\section{Introduction}

Stroke is one of the leading causes of long-term adult disability, affecting approximately 120,000 people each year in Hungary. Early recovery and rehabilitation can improve motor functions and mental state. Physical conditions post-stroke include weakness, numbness, and stiffness. After the stroke, experiencing emotional changes can occur due to natural responses or changes caused by physical effects of the brain. Cognition problems: after stroke, the process of thinking, remembering and recognizing things called (cognition) can become challenging. In our outpatient clinic, we mainly see patients suffering from after-stroke symptoms, as muscle stiffness, hemiplegic limbs, and motor (or rarely sensory) aphasia, depression of mood, epileptic seizures, and difficulties in the daily practice of life. This pathogenesis limits the ability to work, inhibits patients'self-management, and significantly reduces their quality of life (QoL). Acupuncture has been used in Traditional Chinese Medicine to relieve pain and cure a variety of diseases for more than 2500 years. Acupuncture has been used for stroke in China and Korea for centuries, but scientific studies on this topic have only recently started to emerge. Some but not all of these trials have suggested a positive effect on recovery. There are numerous reports in the Chinese literature about the efficacy of acupuncture in stroke rehabilitation. However, the result is rarely quantitatively expressed by properly validated measures, and intention to treat analysis was never mentioned. ${ }^{1-3}$ We introduced since 1989 a unique permanent acupuncture method for patients to avoid necessary weekly treatments and provide them to achieve this additional rehabilitation form living far from the clinic., 


\section{Background/description of the investigated therapy}

According to our practice, the rehabilitation actually should start in the hospital as soon as possible after a stroke. The patients who have a stable cardiopulmonary situation, rehabilitation may begin within two days after the stroke has occurred, and should be continued as necessary after release from the hospital, maybe for several years. Depending on the severity of the brain damage, rehabilitation options can be varied.

\section{A permanent care unit controls}

i. A rehabilitation hospital with individualized inpatient therapy

ii. Home therapy

iii. Returning home with outpatient therapy

iv. A long-term care facility that provides therapy and skilled nursing care

v. YNSA (Yamamoto New Scalp Acupuncture System) is most frequently used for neuromusculoskeletal diseases, dysfunctions, and pain that may be either acute or chronic. ${ }^{6-8}$

\section{Topographical anatomy and neural connection}

YNSA acupuncture method is composed of categories: Basic points, Ypsilon points, and Parietal Points. The Basic points are scalp acupuncture points used to treat pain and dysfunction of anatomical body parts that are directly related to the disease and dysfunctional process. Basic points are associated with small elevations or firmness in the subcutaneous tissue of the scalp at prescribed locations. YNSA also uses Ypsilon points, which relate to the 12 classical acupuncture meridians (LU, LI, KI, BL, HT, SI, LR, GB, PC, TB, SP, and ST). Since 1973, the YNSA method started to be applied to treat stroke patients worldwide; in our hospital, (Yamamoto rehabilitation Institute, Budapest) the technique has been used since 1988, which is permanently developed by prof. Yamamoto ${ }^{9,10}$

Indications: neurological diseases, musculoskeletal pains originated from different cause, muscle, tendon stiffness, rehabilitation of stroke, mood disorders

\section{Contraindications}

i. Instable cardio-pulmonary situation

ii. After acute heart attack stage

The cost-benefit ratio indicates YNSA usage as a complementary valuable option to standard conservative therapy. We applied YNSA and parietal points described first by T. Yamamoto, (see Pictures 1. and 2.)

Objectives: The aim of the investigation was, to assess the effects of YNSA on the circulation of the brain, the consecutive ischaemic pain, the changes in trophic disturbances and patient's quality of life - using validated objective and subjective measurements. Our second aim was to observe possible complications and adverse reactions caused by the intervention, to compare our results with similar, previously published studies, to conclude on the applicability of this dry needling method on patients with post-stroke syndrome.

Study design: A prospectively randomized case series was conducted in our outpatient rehabilitation-clinic over 36 months in Yamamoto
Rehabilitation Institute, Budapest, Hungary. Patients got the detailed and clear explanation of the permanent acupuncture intervention and the preceding tests necessary for the study and signed written informed consent. The study was following the principles of the Declaration of Helsinki, with the written approval of the local Research Ethics Committee (under registration number: 257/2016).

\section{Material patients}

\section{Selection of patients}

Under real-life conditions, all self-selected patients were included, if they fulfilled the inclusion criteria and signed their informed consent.

\section{Inclusion criteria}

i. Stroke in past two years

ii. Hemiparalyse, a stiffness of flexor muscles of hand and foot persisted,

iii. Age 18-78 years,

iv. Clear consciousness,

v. Balanced cardio-pulmonary state.

\section{Exclusion criteria}

Imbalance of cardio-respiratory system.

\section{Outcomes and method}

Objective outcomes: Measurement Barthel index and Rivermead scale, before treatment and on day $30,60,90,120,360 .{ }^{11-13}$

Subjective outcomes: Self-evaluation of the patients

i. VAS (14) was used for defining the intensity of pain, movement, mood disorders, QoL Scale $0-10: 9$ to $10=$ no pain, better movement, minimal stiffness; 7to $8=$ mild pain, mild stiffness, barely affecting everyday life; 4 to $6=$ moderate pain: significantly bothers everyday life, mild depression; 1 to $3=$ severe pain: paralyze, hinders everyday life, deep depression.

ii. SF-36 questionnaire usage for monitoring changes in quality of life. ${ }^{15-19}$

\section{Clinical setting/treatment}

Diagnostics were performed before entering the study: typical complains and symptoms of the disease. Instrumental investigations such as MRI, CT were performed during the previous conventional treatment. Patients continued to take other previously given adjusted medications and gymnastic exercises since they had no influence on our monitoring.

\section{Steps of the investigation}

i. Anamnesis

ii. Physical examination

iii. Measurements before the intervention (Barthel index, Rivermead index, VAS, SF 36 questionnaire)

iv. Intervention: YNSA and parietal points as a permanent form of acupuncture.

v. Measurements after the intervention 
vi. We observed every patient for about $1 / 2$ hour after the intervention. As their vital parameters were stable, they were allowed to leave our ambulance.

vii. The intervention was repeated once a month, that is six times altogether.

\section{Method}

520 patients had been enrolled (260 to receive acupuncture treatment and 260 for control. The course of apply has been between 2013 and 2017, after. 260 (50\%) consecutive patients admitted to the stroke rehabilitation unit were included in the study after informed consent (Figure 1). Inclusion criteria were the following: (1) patients with hemorrhagic or ischemic stroke, (2) admission within 6 weeks of stroke, (3) patients should have movement coordination problems including muscular atrophy, central paralysis, and also myasthenia symptoms, and (4) the degree of brain damage of stroke patients did not exceed $30 \%$ of damaged brain tissue. The last statement is important, because when the brain damage exceeds $30 \%$, usually the dry needling treatment would not be successful. ${ }^{20-23}$

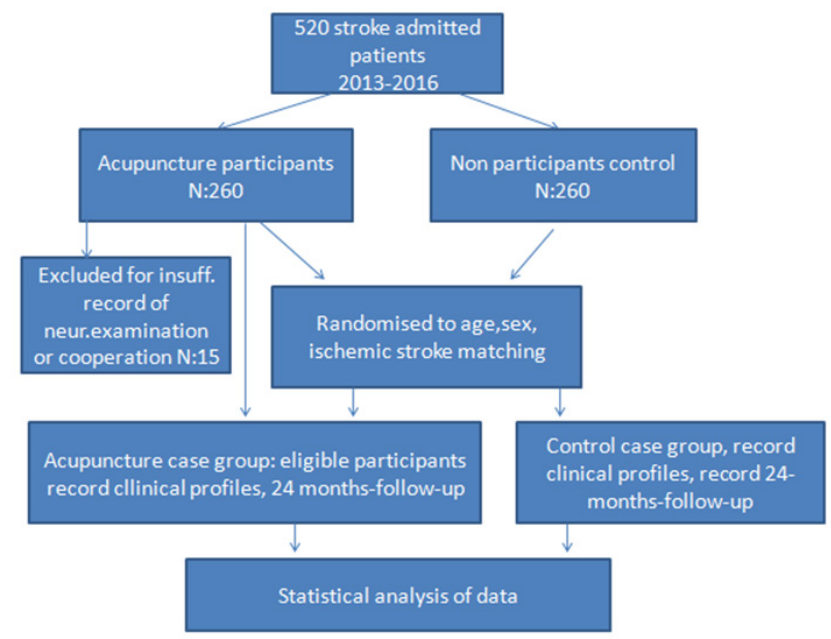

Figure I The average follows up time was two years after treatment.

The size and anatomical location of stroke lesions are evaluated by magnetic resonance imaging using subtraction lesion analysis.

\section{Exclusion criteria were the following}

i. No motor deficit,

ii. Hemodynamic instability,

iii. History of dementia, and

iv. Inability to give consent because of impaired cognition or receptive aphasia.

v. All the selected patients went under the special protocol of rehabilitation program using the Hungarian standard rehabilitation protocol. ${ }^{24}$ We have not enrolled patients in sham-acupuncture therapy (Table 1).

\section{Steps of the intervention}

i. Patient lying with slightly outstretched neck

ii. With the finger, we palpate the small areas on scull to find any softer/ harder area which corresponds YNSA points according to YNSA map (Figures 2) (Figure 3). iii. Over the lying finger, a fine $3-4 \mathrm{~cm}$ long needle (size 12) is pierced dorsally through the skin. After getting the sensitive area contact, we push the mandrine towards, so the absorbent monofilament goes to tissue. After withdrawing the needles with mandrine, we save the insertion.

iv. As a result: a few minutes later can be seen the effect, as improved movement ability, which lasts $2-3$ weeks.

v. The next intervention follows the same procedure

Table I Demographic and Clinival Characteristics of treated patients by Group

\begin{tabular}{lll}
\hline Male Gender \% & I45 & I I 5 \\
\hline Mean age in years (SD) & $59.8(9.6)$ & 58.6 (I0.4) \\
Current smoking, \% & 86 & 60 \\
High school or higher educational level, \% & 90 & 56 \\
Depression (clinical diagnosis), \% & 25 & 24 \\
Very intense pain at presentation, \% & 50 & 45 \\
Lesion of brain tissue, \% & 15.8 & 17.1 \\
Ischemic stroke vs all, \% & 92 & 92 \\
\hline
\end{tabular}
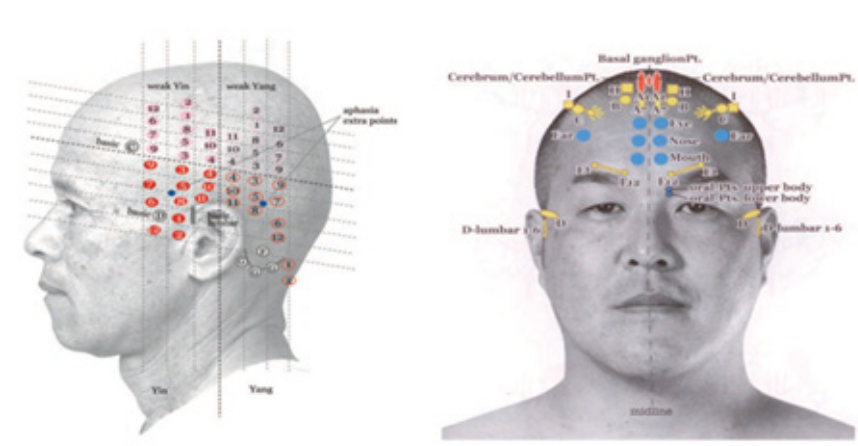

Figure 2 YNSA MAP,Ypsilon and Basic points on scalp.

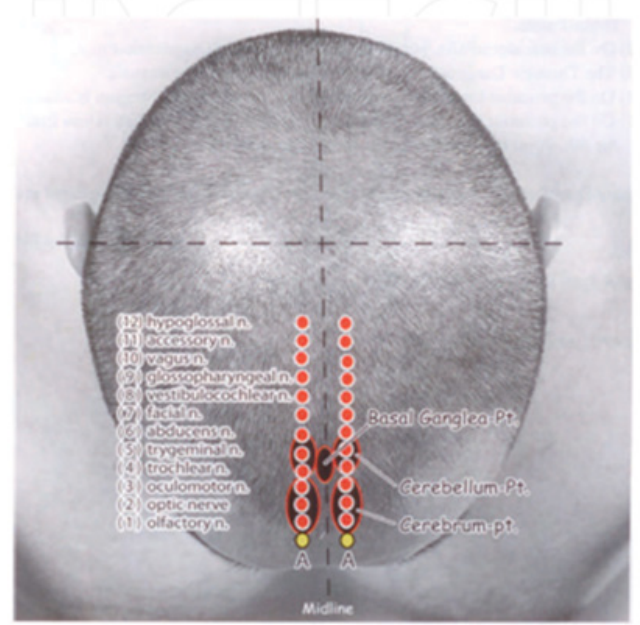

Figure 3 YNSA MAP, parietal points for nerves.

Traditional acupuncture therapy (dry needling) is usually done by the insertion of thin metal needles (Gauge 30) to the acupoints, and this is followed by the slow manual twisting action of the acupuncturist. The needles, once correctly inserted into the acupoints, 
can also be stimulated electrically. The application of needles might even be a noxious stimulus causing autonomic hyperreflexia. Another later developed acupuncture method was the YNSA, where we applied small absorbable threads can make continuous biostimulation of acupoints. The treatment period took 12 months, with monthly one session. We compared the result before and after the treatment. The last follow up was performed on day 365 to detect - not just temporary but-durable effects of the intervention.

\section{Statistical evaluation}

Change in pain intensity was calculated by the $\chi 2$ test. Changes before and after treatment in each dimension of the SF-36 questionnaire were performed by Wilcoxon's test. Data were recorded and analyzed using the SYSTAT 10 program package, according to previous studies and the SF-36 manual. The correlation-calculation method and the 'Excel and built-in functions' software package were used for data analysis. Level of $\mathrm{p}<0.05$ was determined as significant.

\section{Results}

\section{Regarding baseline characteristics}

Data of 520 examined and 260 treated patients were collected. All other patients seen at this time in the outpatient pain-clinic with same symptoms did not fulfill the inclusion criteria or were not willing to participate in the study. Distribution of the investigated patients was: 145 male (mean age 59.0 of years) and 115 female (mean age 41.2 ofyears) patients were detected (Table 1).

The primary outcome was the change in Barthel ADL score. Secondary outcome measures included National Institutes of Health Stroke Scale score, motoricity index, quality of life (EQ-5D [EuroQoL-5 Dimensional form] and EQ-VAS [EuroQoL-Visual Analog Scale]),

\section{Regarding outcomes}

The average follows up time was two years after treatment. 15 dropouts were detected (Figure 1).

\section{Objective results}

i. Aggregated changes in the VAS and QoL showed significant improvement $(\mathrm{p}=0.002)$ (Figure 4).

ii. Aggregated changes in the parameters showed significant improvement $(\mathrm{p}<0.05)$.

iii. In Barthel index, in Rivermead Scale (Figure 4).

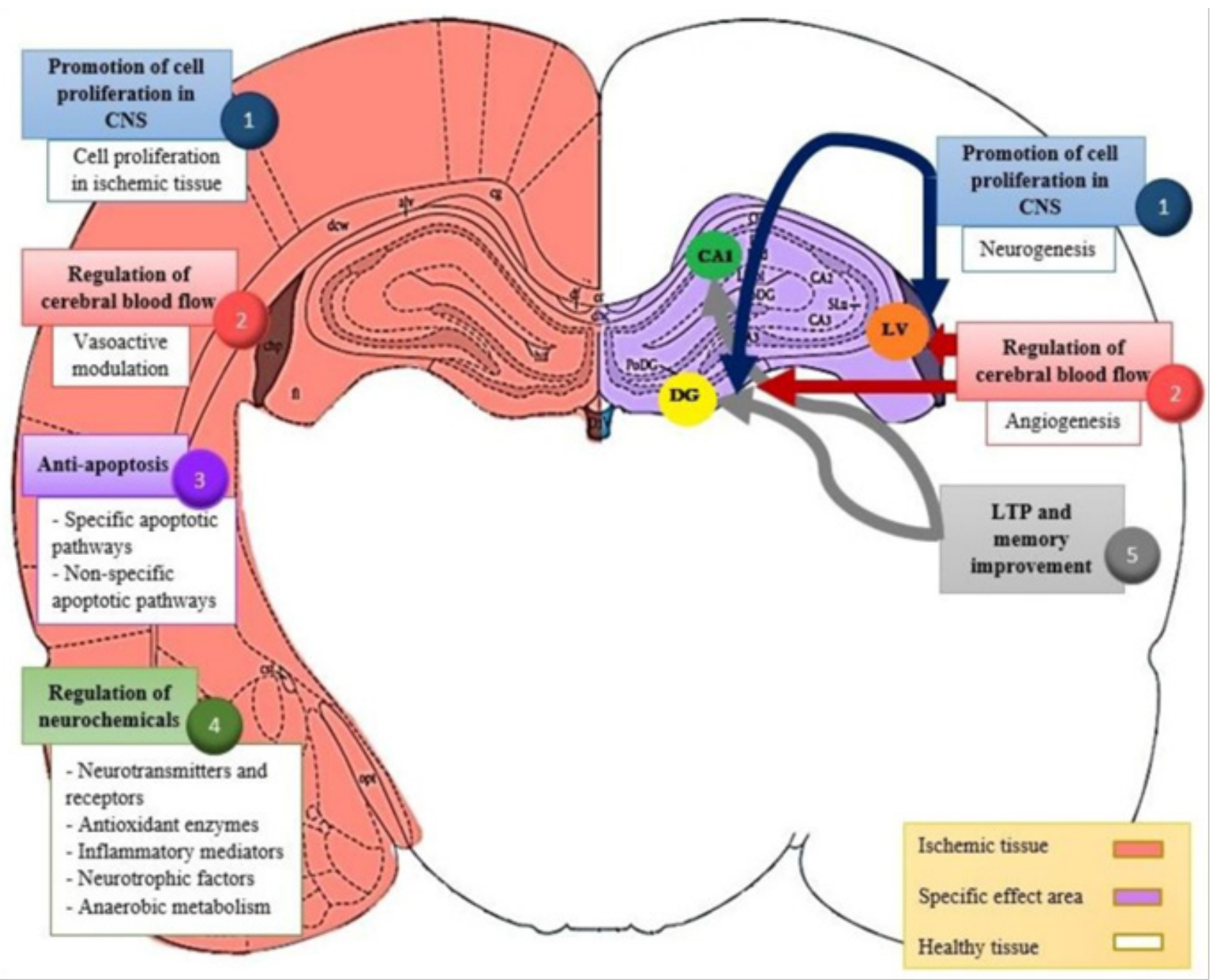

Figure 4 Demaged and healthy functions of CNS.

Citation: Molnár I, Máté A, Szőke H, et al. Effects of permanent acupuncture of YNSA (Yamamoto new scalp acupuncture) on quality of life in patients suffering from post-stroke syndrome. Int J Complement Alt Med. 2018;I (I):97-104. DOI: 10.15406/ijcam.2018.1 I.00356 


\section{Subjective outcomes:}

i. VAS values: 49 patients had pain value of 9 , and 54 patients had pain value of 7 before treatment. After the procedure 12 patients marked worth 5,18 patients marked pain value 2 , and 14 patients scored pain value 0 (Figure 2). So changes in VAS values showed significant improvement $(\mathrm{p}=0.001)$.

ii. In the dimensions of the quality of life, the aggregated change of SF-36 questionnaire also showed a significant improvement $(\mathrm{p}=0.005)$. Table 2 shows there was no relevant difference between male and female patients concerning the dimension of changes in the averaged values, but the age paid the relative big role in hope and imagination of the future of involved patients. Patients imaginations with younger age were more positive as elder's.

Adverse reactions: No lasting complications were detected in any case.

Table 2 Pretreatment and posttreatment scores of the SF-36 questionnaire

\begin{tabular}{|c|c|c|c|c|c|c|c|}
\hline Sf-36 dimensions & Number of cases & Two-Sided probability & Median & Mean & $\begin{array}{l}\text { Standard } \\
\text { deviation }\end{array}$ & $25^{\text {th }}$ percentile & $75^{\text {th }}$ percentile \\
\hline \multirow[t]{2}{*}{ FP-I } & & & 72.500 & 67.917 & 17.896 & 57.5 & 85 \\
\hline & 245 & $\mathrm{p}=0.005^{*}$ & & & & & \\
\hline FP-2 & & & 90.000 & 86.250 & 17.854 & 83.75 & 96.25 \\
\hline \multirow[t]{2}{*}{ RP-I } & & & 25.000 & 29.167 & 33.428 & 0 & 0 \\
\hline & 245 & $\mathrm{p}=0.007^{*}$ & & & & & \\
\hline RP-2 & & & 100.000 & 79.167 & 39.648 & 87.5 & 100 \\
\hline \multirow[t]{2}{*}{ BP-I } & & & 26.500 & 23.750 & 13.897 & 16 & 34 \\
\hline & 245 & $\mathrm{p}=0.002^{*}$ & & & & & \\
\hline BP-2 & & & 70.000 & 69.750 & 14.759 & 62 & 83 \\
\hline \multirow[t]{2}{*}{$\mathrm{GH}-\mathrm{I}$} & & & 22.500 & 22.917 & 18.148 & 8.75 & 31.25 \\
\hline & 245 & $\mathrm{p}=0.01 \mathrm{I}^{*}$ & & & & & \\
\hline $\mathrm{GH}-2$ & & & 26.000 & 28.083 & 19.033 & 15.25 & 37 \\
\hline \multirow[t]{2}{*}{ VT-I } & & & 42.500 & 41.250 & 20.794 & 30 & 51.25 \\
\hline & 245 & $\mathrm{p}=0.01 \mathrm{I} *$ & & & & & \\
\hline VT-2 & & & 57.500 & 55.833 & 22.139 & 48.75 & 68.75 \\
\hline \multirow[t]{2}{*}{ SF-I } & & & 50.000 & 40.583 & 25.681 & 25 & 50 \\
\hline & 245 & $p=0.004^{*}$ & & & & & \\
\hline SF-2 & & & 75.000 & 80.250 & $|8.06|$ & 75 & 100 \\
\hline \multirow[t]{2}{*}{ RE-I } & & & 16.500 & 38.833 & 46.789 & 0 & 100 \\
\hline & 245 & $\mathrm{p}=0.038^{*}$ & & & & & \\
\hline RE-2 & & & 100.000 & 75.000 & 45.227 & 75 & 100 \\
\hline \multirow[t]{2}{*}{ MH-I } & & & 50.000 & 48.667 & 17.042 & 39 & 60 \\
\hline & 245 & $\mathrm{p}=0.005^{*}$ & & & & & \\
\hline $\mathrm{MH}-2$ & & & 60.000 & 60.667 & 15.144 & 51 & 73 \\
\hline
\end{tabular}

I=before therapy, $2=$ after therapy; $*$ indicates significant difference.

FP, physical functioning; PHP, physical health problems; RP, physical role functioning; BP, bodily pain; GH, general health perceptions; VT, vitality; Energy/Fatique; SF, social role functioning; RE, emotional role Functioning, emotional health problems; $\mathrm{MH}$, mental health; Emotional well-being 
Table 3 Changes of Barthel index, Rivermead index, and visual analogue scale (VAS) in acupuncture (black columns) and control (white columns) group.
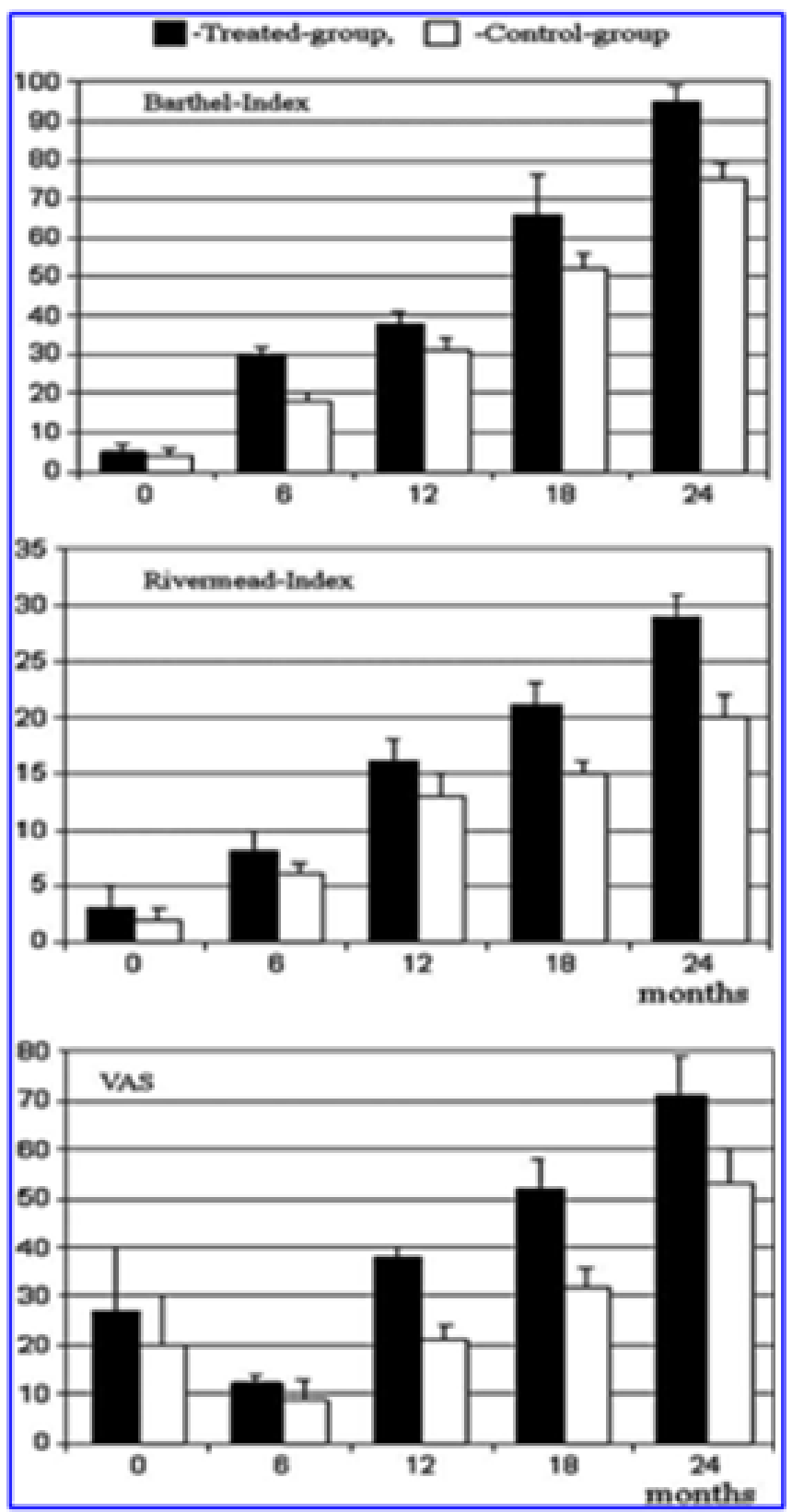

\section{Discussion}

Several previous stroke disease-specific studies confirm that additional acupuncture treatment of post-stroke patients is an unsuccessful treatment. We aimed to compare our results with the outcomes of other similar studies lately published where the outcome of treatment elevated the movement of patients and QoL. $(25,26)$ However, we could not find any case reports that aimed to improve circulation of the limbs in patients with post-stroke syndrome by treating with dry needling, and that followed up changes in quality of life. According to ethical and professional reasons we create a control group for patients with same problems. Bias was taken into account and minimized. This study aimed to examine the long-term effects of repeated embedded YNSA treatment on balance and activities of daily living in post-acute stroke patients compared with the no- acupuncture group. "What is the truth about acupuncture in stroke rehabilitation? In the authors' opinion at least two things are important to determine the efficacy of the acupuncture treatment in stroke rehabilitation. The most important is to enhance the life quality of the stroke patients during and after the acupuncture treatment. The second thing is to find the possible link between the acupuncture treatment and the cellular mechanisms that activated during the recovery period of stroke. Previous studies have reported the ineffectiveness of sensory stimulation by acupuncture or transcutaneous electrical nerve stimulation, functional electrical stimulation, electromyographic feedback, force feedback or body weight supported treadmill training on balance in patients with stroke. Recent evidence has shown that impairment-focused programs (biofeedback, electric stimulation, muscle strengthening) failed to generate functional improvements whereas therapies that administered functional training improved activity levels. ${ }^{26}$ Unfortunately, there are only a few previous data published about the YNSA treatment in stroke survivors. However, in the current study, the used BI, the RMI, SP-36, and the VAS were applied to determine the changes of life qualities of participants. The patients were followed in a 2-year period after the stroke. All of the four parameters were increased in the 24 months follow-up period in the acupuncture and control group; however, the changes were significantly greater in the acupuncture group than in the control group. The data suggest that the YNSA method is a useful complementary intervention to treat the stroke patients and enhance the quality of life. Using BI, RMI, SP-36, and VAS for the motor-status evaluation, the results showed that YNSA is effective in patients with stroke (ischemic and hemorrhagic) for motor recovery. To the author's knowledge, this is the reproduction of our first study on this topic, to demonstrate that YNSA has beneficial effects on body motor performance in patients with stroke. ${ }^{27}$ Regarding functional improvement, both the study group and the control group showed improvement in functional performance at follow-up assessments. However, there was a tendency for the study group to be superior to the control group in the total scores at follow-up stages, and at the end of the follow-up period, it did reach statistical significance. However, the link still must be found between the acupuncture and the cellular changes after stroke. Some evidence suggests that the acupuncture stimulation with a needle has some cellular and tissue effects and that the acupuncture can enhance the release of transmitters that can facilitate angio-and neurogenesis in the central neural system. ${ }^{28}$ On a cellular level, the phenomenology of neural repair after stroke has been defined and unique regenerative environments in the post-stroke brain identified. These data suggest that acupuncture facilitates some molecular and cellular mechanisms that have an important role in the angiogenesis, neurogenesis and tonic neuronal inhibition in brain tissue. ${ }^{29-33}$ This it can be concluded that acupuncture, especially the YNSA, is a useful complementary tool to facilitate stroke rehabilitation.

\section{Conclusion}

Hemiparalyse, depression and chronic pain cause a decline in the quality of life. Although analgesia is obviously a symptomatic therapy, our results showed, that - independently from the underlying cause - the permanent technique of acupuncture specialized to YNSA and scalp points can achieve a durable improvement in symptoms and quality of life of patients. By treating the scalp acupuncture points, circulation and innervations of the hands and legs can be improved significantly is already proved. ${ }^{30-32}$ The actual question today still the follow: does acupuncture work to help stroke victims improve? More studies involving thousands of patients have been published in Eastern, and 2 of 3 studies from the Western part of World, 
demonstrated a significant help. These studies indicate that patients get well faster, perform better in self-care, require less nursing and rehabilitation therapy, and use less healthcare cover. From the available evidence, acupuncture and a unique permanent form of YNSA may have beneficial effects on improving dependency, global neurological deficiency, and some specific neurological impairments for people with stroke in the convalescent stage, with no obvious serious adverse events. ${ }^{34-36}$ (3.picture) Further investigations can be done with a larger number and longer follow-up of patients. These data suggest that the use of YNSA therapy with embedded threads after stroke can contribute to significant neurologic and functional recoveries. We proved higher positive effect in patients with smaller damaged brain area, (under 30\%) earlier starting the treatment after stroke and younger age.

\section{There are several limitations of this study}

Because we have not enrolled patients for sham control, it is not known how much the YNSA intervention contributed to the improvement of subjects in the acupuncture group be - but it is not known how much of the improvement was due to the placebo effect. The placebo effect an additional factor for improving mental state. Further data exploration suggested that these complete case analyses are likely to provide valid statistical interferences.

\section{Acknowledgement}

First of all many thanks to professor Yamamoto to introduce and teach us this system. Acupuncture according to YNSA, belongs to the CAM methods in more European countries. ${ }^{37}$ (CAMbrella PanEuropean project, 2010-2012), is one of its advanced methods too. In some countries (Austria, Germany, Hungary, Czech R, Slovakia, Greek) it is accepted official CAM methods for medical doctors only, providing better QoL the patient for different diseases which are involved in its indication. Pecs University CAM Department has organized more official courses for MDs according to Yamamoto News Scalp Acupuncture.

\section{Conflict of interest}

The authors declare no conflict of interest in this study.

\section{References}

1. Ernst E. The need for scientific rigor in studies of complementary and alternative medicine. Am J Public Health. 2004;94(7):1074-1075.

2. Chavez LM, Huang SS, MacDonald I, et al. Mechanisms of acupuncture therapy in ischemic stroke rehabilitation: a literature review of basic studies. Int J Mol Sci. 2017;18(11):2270.

3. Li X, Wang Q. Acupuncture therapy for stroke patients. Int Rev Neurobiol. 2013;111:159-179.

4. Hegyi G, Szigeti GP. Rehabilitation of stroke patients using Yamamoto New Scalp Acupuncture:a pilot study. J Altern Complement Med. 2012;18(10):971-977.

5. Hegyi G, Pfeiffenroth A, Csutortoki K. Hungarian military use of acupuncture: recent clinical trials and accumulated survey data medical acupuncture. 2012;24(3):152-155.

6. Schockert T, Schnitker R, Boroojerdi B. Cortical activation by Yamamoto new scalp acupuncture (YNSA) in the treatment of stroke patients-a sham-controlled Study aided by functional magnetic resonance imaging (fMRI). Dt Zeitschr f Akup. 2012;1:21-29.

7. Schockert T, Beissner F. Neurophysiological Correlates of the Effect of YNSA for Patients with Chronic Pain of the Locomotor System Basic YNSA Research by Means of PET-CT. Deutsch Ztschr f Akup. 2010;2:8-13.
8. Schockert T, Schnitker R, Boroojerdi B, et al. Cortical activation by yamamoto new scalp acupuncture (YNSA) in the treatment of stroke patients - a sham-controlled study using functional magnetic resonance imaging (fMRI). Acupunct Med. 2010;4:212-214.

9. Yamamoto T, Yamamoto $\mathrm{H}$, Yamamoto MM. Yamamoto Neue Scha"delakupunktur. Bad Ko tzting: VGM; 2005.

10. Schockert T. YNSA Individualtherapie durch Halsdiagnos-tik. Komplement Integr Med. 2010;10:8-10.

11. Mahoney FI, Barthel DW. Functional evaluation: the barthel index. $M d$ State Med J. 1965;14:61-65.

12. Granger CV, Dewis LS, Peters NC. Stroke rehabilita- tion:Analysis of repeated Barthel index measures. Arch Phys Med Rehabil. 1979;60(1):14-17.

13. Wade DT, Collin C. The Barthel ADL Index:A standard measure of physical disability? Int Disabil Stud. 1988;10:64-67.

14. Ahlsio B, Britton M, Murray V, et al. VAS disablement and quality of life after stroke. Stroke. 1984;15(5):886-890.

15. Hawker GA, Mian S, Kendzerska T, et al. Measures of adult pain: visual analog scale for pain (VAS Pain), numeric rating scale for pain(NRS Pain), McGill pain questionnaire (MPQ), short-form mcgill pain questionnaire (SF-MPQ), chronic pain grade scale (CPGS), short form-36 bodily pain scale (SF-36 BPS), and measure of intermittent and constant osteoarthritis pain (ICOAP). Arthritis Care Res (Hoboken). 2011;11:S240-252.

16. Bunevicius A. Reliability and validity of the SF-36 Health Survey Questionnairein patients with brain tumors:a cross-sectional study. Health Qual Life Outcomes. 2017;15(1):92.

17. Salim S, Yamin M, Alwi I, et al. Validity and reliability of the indonesian version of SF-36 quality of life questionnaire on patients with permanent pacemakers. Acta Med Indones. 2017;49(1):10-16.

18. Ware J. SF-36 Health survey update. Spine. 2000;25(24):3130-3139.

19. Naeser M, Martin PI, Baker EH, et al. Overt propositional speech in chronic nonfluent aphasia studied with the dynamic susceptibility contrast fMRI method. Neuroimage. 2004;22(1):29-41.

20. Naeser, Baker EH, Doron K, et al. Test-retest reliability of fMRI during nonverbal semantic decisions in moderate-severe nonfluent aphasia patients. Behav Neurol. 2004;15(3-4):87-97.

21. Van Peppen RPS, Kwakel G, Wood-Dauphinee S, et al. The impact of physical therapy on functional outcome after stroke:What's the evidence? Clin Rehabil. 2004;18:833-862.

22. Alexander LD, Black SE, Gao F, et al. Correlating lesion size and location to deficits after ischemic stroke: the influence of accounting for altered peri-necrotic tissue and incidental si- lent infarcts. Behav Brain Funct. 2010;6:6-15.

23. Endres M, Nyary I, Banhidi M, et al. stroke rehabilitation:A method and evaluation. Int J Rehabil Res. 1990;13(3):225-236.

24. Park J, Hopwood V, White AR, et al. Effectiveness of acupuncture for stroke:A systematic review. J Neurol. 2001;248:558-563.

25. Sze FK, Wong E, Or KK, et al. Does acupuncture improve motor recovery after stroke? A meta-analysis of randomized controlled trials. Stroke. 2002;33:2604-2619.

26. Bai L, Tao Y, Wang D, et al. Acupuncture induces time-dependent remodelling brain network on the stable somatosensory first-ever stroke patients: combining diffusion tensor and functional MR imaging. Evidence-Based Complementary and Alternative Medicine. 2014. p. $1-7$.

27. Park SU1, Shin AS, Jahng GH, et al. Effects of scalp acupuncture versus upper and lower limb acupuncture on signal activation of blood oxygen level dependent (BOLD) fMRI of the brain and somatosensory cortex. $J$ Altern Complement Med. 2009;15(11):1193-1200. 
28. Lynch MA. Long-term potentiating and memory. Physiol Rev. 2004;84(1):87-136.

29. Chavez LM, Huang SS, MacDonald I, et al. Mechanisms of acupuncture therapy in ischemic stroke rehabilitation: a literature review of basic studies. Int J Mol Sci. 2017;18(11):2270.

30. Li X, Wang Q. Acupuncture therapy for stroke patients. Int Rev Neurobiol. 2013;111:159-179.

31. Carmichael ST. Themes and strategies for studying the biology of stroke recovery in the poststroke epoch. Stroke. 2008;39(4):1380-1388.

32. Ding Y, Yan Q, Ruan JW, et al. Electro-acupuncture pro- motes survival, differentiation of the bone marrow mesen- chymal stem cells as well as functional recovery in the spinal cord-transected rats. BMC Neurosci. $2009 ; 10: 35$
33. Cooper DMF, Mons N, Karpan JW. Adenylyl cyclases and the interaction between calcium and cAMP signaling. Nat- ure. 1995;374(6521):421424.

34. Ghosh A, Carnahan J, Greenberg ME. Requirement for BDNF in activitydependent survival of cortical neurons. Science. 1994;263(5153):16181623.

35. Hansen MR, Zha XM, Bok J, et al. Multiple distinct signal pathways, including an autocrine neurotrophic mechanism, contribute to the survival-promoting effect of depolarization on spiral ganglion neurons in vitro. $J$ Neurosci. 2001;21(7):2256-2267.

36. Fu LW, Longhurst JC. Electroacupuncture modulates vlPAG release of GABA through presynaptic cannabinoid CB1 re- ceptors. J Appl Physiol. 2009;106(6):1800-1809.

37. www.cambrella.eu 